\title{
Decay to zero of matrix coefficients at Adjoint infinity
}

by

Scot Adams

\section{Introduction}

The main theorems below are Theorem 9 and Theorem 11. As far as I know, Theorem 9 represents a slight improvement over what currently appears in the literature, and gives a fairly easy proof of Theorem 11 which is due to R. Howe and C. Moore [4]. In the semisimple case, the Howe-Moore result follows from [6] or [7].

The proofs appearing here are relatively elementary and some readers will recognize the influence of R. Ellis and M. Nerurkar [2]. What may be less evident is the connection to N. Kowalsky's work [5] on dynamics on Lorentz manifolds: Lemma 1 below is a unitary analogue of the fact that an expansive Adjoint action can result in much of the Lie algebra being lightlike. In Hilbert space, the situation is even nicer: an isotropic vector must equal zero, so we get that the Lie algebra of the stabilizer contains all "Kowalsky" vectors for the Adjoint sequence, and not just a codimension one subspace. Moreover, much of the rest of proof of Theorem 9 also uses ideas that were originally developed to describe effectively the collection of simply connected Lie groups admitting an orbit nonproper action on a connected Lorentz manifold. Thus the debt to Kowalsky is significant.

In this note, we assume some familiarity with Lie theory and basic unitary representation theory. The exposition should otherwise be self-contained.

Please send comments, suggestions or questions to adams@math.umn.edu.

Throughout, "Lie group" means " $C^{\infty}$ real Lie group", "Lie algebra" means "real Lie algebra" and "Hilbert space" means "complex Hilbert space". Lie groups are denoted by capital Roman letters and, for any Lie group, its Lie algebra is denoted, without comment, by the corresponding small letter in the fraktur font. The sesquilinear form on a Hilbert space is denoted $\langle\cdot, \cdot\rangle$. Convergence in the weak topology on a Hilbert space is denoted with $\rightarrow$, so $v_{i} \rightarrow v$ in a Hilbert space $\mathcal{H}$ means: for all $w \in \mathcal{H},\left\langle v_{i}, w\right\rangle \rightarrow\langle v, w\rangle$. The group of bounded operators on $\mathcal{H}$ is denoted $B(\mathcal{H})$. The unitary group of a Hilbert space $\mathcal{H}$ is denoted $U(\mathcal{H})$. The weak-operator and strong-operator topologies agree on $U(\mathcal{H})$, and $U(\mathcal{H})$ is given this topology. Unitary representations are always assumed continuous in the weak-operator topology (or, equivalently, in the strong-operator topology).

I do not plan to publish this note.

\section{Preliminary dynamical results}

Let $G$ be a Lie group acting by a unitary representation on a Hilbert space $\mathcal{H}$. Let $w \in \mathcal{H}$ and let $\mathfrak{m}$ be the Lie algebra of $\operatorname{Stab}_{G}(w)$. 
Lemma 1: Let $U_{i} \rightarrow 0$ in $\mathfrak{g}$. Let $g_{i}$ be a sequence in $G$. Assume $\left(\operatorname{Ad} g_{i}\right) U_{i} \rightarrow X$ in $\mathfrak{g}$. Let $v \in \mathcal{H}$ and assume that $g_{i} v \rightarrow w$ in $\mathcal{H}$. Then $X \in \mathfrak{m}$.

Proof: Fix $t \in \mathbb{R}$ and let $h:=\exp (t X)$. We wish to show that $h w=w$.

As $\exp \left(t U_{i}\right) \rightarrow 1_{G}$ in $G$, we get $\left(\exp \left(t U_{i}\right)\right) v \rightarrow v$ in $\mathcal{H}$. So, for all $x \in \mathcal{H}$, since $\left\{g_{i}^{-1} x\right\}$ is norm-bounded in $\mathcal{H}$, Cauchy-Schwartz yields: $\left\langle\left[\left(\exp \left(t U_{i}\right)\right) v\right]-v, g_{i}^{-1} x\right\rangle \rightarrow 0$; then

$$
\left\langle g_{i}\left(\exp \left(t U_{i}\right)\right) v, x\right\rangle-\left\langle g_{i} v, x\right\rangle \rightarrow 0
$$

Moreover, as $g_{i} v \rightarrow w$, we see, for all $x \in \mathcal{H}$, that

$$
\left\langle g_{i} v, x\right\rangle-\langle w, x\rangle \rightarrow 0
$$

Adding the last two displayed limits, for all $x \in \mathcal{H}$, we have $\left\langle g_{i}\left(\exp \left(t U_{i}\right)\right) v, x\right\rangle-\langle w, x\rangle \rightarrow 0$. That is, $g_{i}\left(\exp \left(t U_{i}\right)\right) v \rightarrow w$ in $\mathcal{H}$.

For all $i$, let $X_{i}:=\left(\operatorname{Ad} g_{i}\right) U_{i}$ and let $h_{i}:=\exp \left(t X_{i}\right)$. Then, for all $i$, we have $g_{i}\left(\exp \left(t U_{i}\right)\right) g_{i}^{-1}=h_{i}$, so $h_{i} g_{i}=g_{i}\left(\exp \left(t U_{i}\right)\right)$. Then $h_{i} g_{i} v=g_{i}\left(\exp \left(t U_{i}\right)\right) v \rightarrow w$ in $\mathcal{H}$.

We have $X_{i} \rightarrow X$ in $\mathfrak{g}$, and so $h_{i} \rightarrow h$ in $G$. For all $x \in \mathcal{H}$, since $h_{i}^{-1} x \rightarrow h^{-1} x$ and since $\left\{g_{i} v\right\}$ is norm-bounded in $\mathcal{H}$, Cauchy-Schwartz gives: $\left\langle g_{i} v, h_{i}^{-1} x-h^{-1} x\right\rangle \rightarrow 0$; then

$$
\left\langle h_{i} g_{i} v, x\right\rangle-\left\langle h g_{i} v, x\right\rangle \rightarrow 0
$$

Since $g_{i} v \rightarrow w$, it follows, for all $x \in \mathcal{H}$, that $\left\langle g_{i} v, h^{-1} x\right\rangle-\left\langle w, h^{-1} x\right\rangle \rightarrow 0$; then

$$
\left\langle h g_{i} v, x\right\rangle-\langle h w, x\rangle \rightarrow 0
$$

Adding the last two displayed limits, for all $x \in \mathcal{H}$, we have $\left\langle h_{i} g_{i} v, x\right\rangle-\langle h w, x\rangle \rightarrow 0$. That is, $h_{i} g_{i} v \rightarrow h w$. So, recalling that $h_{i} g_{i} v \rightarrow w$, we get $h w=w$. QED

Corollary 2: For all $T \in \mathfrak{m}$, we have $\left(\mathfrak{c}_{\mathfrak{g}}(T)\right) \cap((\operatorname{ad} T) \mathfrak{g}) \subseteq \mathfrak{m}$.

Proof: Let $X \in\left(\mathfrak{c}_{\mathfrak{g}}(T)\right) \cap((\operatorname{ad} T) \mathfrak{g})$. We wish to show that $X \in \mathfrak{m}$.

We have $(\operatorname{ad} T) X=0$ and $X \in(\operatorname{ad} T) \mathfrak{g}$. Choose $S \in \mathfrak{g}$ such that $(\operatorname{ad} T) S=X$. Let $r_{i}$ be a sequence of nonzero real numbers such that $r_{i} \rightarrow+\infty$. For all $i$, let $g_{i}:=\exp \left(r_{i} T\right)$ and let $U_{i}:=S / r_{i}$. Then $U_{i} \rightarrow 0$ in $\mathfrak{g}$. Since $T \in \mathfrak{m}$, it follows, for all $i$, that $g_{i} w=w$.

Since $(\operatorname{ad} T) S=X$ and $(\operatorname{ad} T) X=0$, it follows, for all $i$, that $\left(\operatorname{Ad} g_{i}\right) S=S+r_{i} X$. Then $\left(\operatorname{Ad} g_{i}\right) U_{i}=\left(S / r_{i}\right)+X \rightarrow X$ in $\mathfrak{g}$, so, by Lemma 1 (with $v:=w$ ), we are done. QED

\section{More preliminaries}

Remark 3: Let $\mathfrak{g}$ be a Lie algebra. Let $U_{i} \rightarrow 0$ in $\mathfrak{g}$ and let $X \in \mathfrak{g}$. Let $g_{i}$ be a sequence in $G$. Assume that $\left(\operatorname{Ad} g_{i}\right) U_{i} \rightarrow X$ in $\mathfrak{g}$. Then ad $X: \mathfrak{g} \rightarrow \mathfrak{g}$ is nilpotent. 
Proof: Let $F_{i} \subseteq \mathbb{C}$ be the set of eigenvalues of ad $U_{i}: \mathfrak{g} \rightarrow \mathfrak{g}$. Then, as $U_{i} \rightarrow 0$, it follows that $F_{i} \rightarrow\{0\}$ in the topological space of finite subsets of $\mathbb{C}$. For all $i$, in $\operatorname{End}(\mathfrak{g})$, we have

$$
\operatorname{ad}\left(\left(\operatorname{Ad} g_{i}\right) U_{i}\right)=\left(\operatorname{Ad} g_{i}\right)\left(\operatorname{ad} U_{i}\right)\left(\operatorname{Ad} g_{i}\right)^{-1}
$$

so $F_{i}$ is also the set of eigenvalues of $\operatorname{ad}\left(\left(\operatorname{Ad} g_{i}\right) U_{i}\right): \mathfrak{g} \rightarrow \mathfrak{g}$. Passing to the limit, $\{0\}$ is the set of eigenvalues of $\operatorname{ad} X: \mathfrak{g} \rightarrow \mathfrak{g}$. QED

Remark 4: Let $V$ be a finite-dimensional real vector space.

(i) Let $T_{i}$ be a sequence in $\mathrm{GL}(V)$. Assume that $\left\{T_{i}\right\}$ is not precompact in $\mathrm{GL}(V)$. Then either $\left\{T_{i}\right\}$ or $\left\{T_{i}^{-1}\right\}$ is not precompact in $\operatorname{End}(V)$.

(ii) Let $E_{i}$ be a sequence in $\operatorname{End}(V)$. Assume that $\left\{E_{i}\right\}$ is not precompact in $\operatorname{End}(V)$. Then there exists $v \in V$ such that $E_{i}(v)$ is not precompact in $V$.

Proof: Proof of (i): For all $i$, let $U_{i}:=T_{i} \oplus T_{i}^{-1} \in \mathrm{GL}(V \oplus V)$, so $U_{i}$ is defined by $U_{i}(v, w)=\left(T_{i} v, T_{i}^{-1} w\right)$. Then $\left\{U_{i}\right\}$ is not precompact in $\operatorname{GL}(V \oplus V)$. For all $i$, we have $\operatorname{det}\left(U_{i}\right)=1$, so $\left\{U_{i}\right\}$ is not precompact in $\operatorname{SL}(V \oplus V)$. So, since $\operatorname{SL}(V \oplus V)$ is closed in $\operatorname{End}(V \oplus V)$, we see that $\left\{U_{i}\right\}$ is not precompact in $\operatorname{End}(V \oplus V)$. Then, as $\left\{U_{i}\right\}=\left\{T_{i} \oplus T_{i}^{-1}\right\}$, the result follows. End of proof of $(i)$.

Proof of (ii): Let $d:=\operatorname{dim}(V)$. Let $v_{1}, \ldots, v_{d}$ be a basis for $V$. Let $\psi_{1}, \ldots, \psi_{d}: V \rightarrow \mathbb{R}$ be a basis for the dual space of $V$. For all $i$, let $M_{i}:=\left[\psi_{k}\left(E_{i}\left(v_{j}\right)\right)\right]_{j k}$ be the matrix of $E_{i}$ with respect to the two bases. Then $\left\{M_{i}\right\}_{i}$ is not precompact in $\mathbb{R}^{d \times d}$. Choose $j, k \in\{1, \ldots, d\}$ such that $\left\{\psi_{k}\left(E_{i}\left(v_{j}\right)\right)\right\}_{i}$ is not precompact in $\mathbb{R}$. Then $\left\{E_{i}\left(v_{j}\right)\right\}_{i}$ is not precompact in $V$. Let $v:=v_{j}$. End of proof of $(i i)$. QED

Let $\mathfrak{g}$ be a Lie algebra and let $X, Y, T \in \mathfrak{g}$. We will say that $(X, Y, T)$ is a standard triple for $\mathfrak{g}$ if $[T, X]=2 X,[T, Y]=-2 Y$ and $[X, Y]=T \neq 0$.

Let $G$ be a Lie group and let $X, Y, T \in \mathfrak{g}$. For all $u \in \mathbb{R}$, let

$$
n(u):=\exp (u X), \quad \bar{n}(u):=\exp (u Y), \quad a(u):=\exp (u T)
$$

We will say that $(X, Y, T)$ is a basic triple for $G$ if, for all $\tau \in \mathbb{R}$, for all $\delta \in \mathbb{R} \backslash\{0\}$,

$$
\left[n\left(\frac{e^{\tau}-1}{\delta}\right)\right] \cdot[\bar{n}(\delta)] \cdot\left[n\left(\frac{e^{-\tau}-1}{\delta}\right)\right]=\left[\bar{n}\left(e^{-\tau} \delta\right)\right] \cdot[a(\tau)]
$$

Remark 5: Let $G$ be a Lie group. Then any standard triple for $\mathfrak{g}$ is a basic triple for $G$.

Proof: Let $(X, Y, T)$ be a standard triple for $\mathfrak{g}$. We wish to show that $(X, Y, T)$ is a basic triple for $G$. Let $S:=\mathrm{SL}_{2}(\mathbb{R})$ and let

$$
X_{0}=\left[\begin{array}{ll}
0 & 1 \\
0 & 0
\end{array}\right], \quad Y_{0}=\left[\begin{array}{ll}
0 & 0 \\
1 & 0
\end{array}\right], \quad T_{0}=\left[\begin{array}{cc}
1 & 0 \\
0 & -1
\end{array}\right]
$$


Then, for all $u \in \mathbb{R}$, we have

$$
\exp \left(u X_{0}\right)=\left[\begin{array}{cc}
1 & u \\
0 & 1
\end{array}\right], \quad \exp \left(u Y_{0}\right)=\left[\begin{array}{ll}
1 & 0 \\
u & 1
\end{array}\right], \quad \exp \left(u T_{0}\right)=\left[\begin{array}{cc}
e^{u} & 0 \\
0 & e^{-u}
\end{array}\right]
$$

so straightforward matrix computations prove that $\left(X_{0}, Y_{0}, T_{0}\right)$ is a standard triple for $\mathfrak{s}$ and a basic triple for $S$. Let $\widetilde{S}$ be the universal cover of $S$ and let $p: \widetilde{S} \rightarrow S$ be a covering homomorphism. Then $d p: \widetilde{\mathfrak{s}} \rightarrow \mathfrak{s}$ is an isomorphism of Lie algebras. Let $\widetilde{X}_{0}:=(d p)^{-1}\left(X_{0}\right)$, let $\widetilde{Y}_{0}:=(d p)^{-1}\left(Y_{0}\right)$ and let $\widetilde{T}_{0}:=(d p)^{-1}\left(T_{0}\right)$. Then $\left(\widetilde{X}_{0}, \widetilde{Y}_{0}, \widetilde{T}_{0}\right)$ is a standard triple for $\widetilde{\mathfrak{s}}$ and, by uniquness of liftings across covering maps, $\left(\widetilde{X}_{0}, \widetilde{Y}_{0}, \widetilde{T}_{0}\right)$ is a basic triple for $\widetilde{S}$.

Since $\left(\widetilde{X}_{0}, \widetilde{Y}_{0}, \widetilde{T}_{0}\right)$ is a standard triple for $\widetilde{\mathfrak{s}}$ and $(X, Y, T)$ is a standard triple for $\mathfrak{g}$, there is a homomorphism of Lie algebras $\phi: \widetilde{\mathfrak{s}} \rightarrow \mathfrak{g}$ such that $\phi\left(\tilde{X}_{0}\right)=X, \phi\left(\tilde{Y}_{0}\right)=Y$ and $\phi\left(\tilde{T}_{0}\right)=T$. By the Monodromy Theorem (see Theorem 2.7.5, on p. 71 of [8]), there is a Lie group homomorphism $f: \widetilde{S} \rightarrow G$ such that $d f=\phi$. Then, by naturality of the exponential map, we see that $(X, Y, T)$ is a basic triple for $G$. QED

Proposition 6: Let $S$ be a connected simple Lie group acting on a Hilbert space $\mathcal{H}$ by a unitary representation. Let $w \in \mathcal{H}$. Let $\mathfrak{m}$ be the Lie algebra of $\operatorname{Stab}_{S}(w)$. Assume $\exists X \in \mathfrak{m} \backslash\{0\}$ such that ad $X: \mathfrak{n} \rightarrow \mathfrak{n}$ is nilpotent. Then $S$ fixes $w$.

Proof: By Jacobson-Morozov (see, e.g., Theorem 7.4 of Chapter IX on p. 432 of [3]), choose $Y, T \in \mathfrak{s}$ such that $(X, Y, T)$ is a standard triple for $\mathfrak{s}$. Then $T \neq 0$. For all $u \in \mathbb{R}$, let

$$
n(u):=\exp (u X), \quad \bar{n}(u):=\exp (u Y), \quad a(u):=\exp (u T) .
$$

Let $A:=\{a(u)\}_{u \in \mathbb{R}}$ be the image of $a: \mathbb{R} \rightarrow S$.

Claim $\alpha: w$ is $A$-invariant. Proof of Claim $\alpha$ : Let $N:=\{n(u)\}_{u \in \mathbb{R}}$ be the image of $n: \mathbb{R} \rightarrow S$. Since $X \in \mathfrak{m}$, we see that $w$ is $N$-invariant.

Define $f: S \rightarrow \mathbb{R}$ by $f(s)=\operatorname{Re}(\langle s w, w\rangle)$. For all $s \in S$, and all $p, q \in N$, we have $\langle p s q w, w\rangle=\left\langle s(q w), p^{-1} w\right\rangle=\langle s w, w\rangle$, so $f(p s q)=f(g)$. That is, $f$ is bi-invariant under $N$.

Let $a_{0} \in A$. We wish to show that $a_{0} w=w$. We have $f\left(1_{S}\right)=\|w\|^{2}$ and

$$
2\left(f\left(a_{0}\right)\right)=-\left\|a_{0} w-w\right\|^{2}+\left\|a_{0} w\right\|^{2}+\|w\|^{2}=-\left\|a_{0} w-w\right\|^{2}+2\|w\|^{2},
$$

so $2\left(f\left(a_{0}\right)\right)-2\left(f\left(1_{S}\right)\right)=-\left\|a_{0} w-w\right\|^{2}$. It therefore suffices to prove $f\left(a_{0}\right)=f\left(1_{S}\right)$.

Fix $\tau \in \mathbb{R}$ such that $a_{0}=a(\tau)$. Let $\delta_{i}$ be a sequence of nonzero real numbers such that $\delta_{i} \rightarrow 0$ in $\mathbb{R}$. For all $i$, let

$$
n_{i}:=n\left(\frac{e^{\tau}-1}{\delta_{i}}\right), \quad \bar{n}_{i}:=\bar{n}\left(\delta_{i}\right), \quad n_{i}^{\prime}:=n\left(\frac{e^{-\tau}-1}{\delta_{i}}\right) .
$$

By Remark 5, since $(X, Y, T)$ is a standard triple for $\mathfrak{s},(X, Y, T)$ is a basic triple for $S$. Then, for all $i$, we have $n_{i} \bar{n}_{i} n_{i}^{\prime}=\left[\bar{n}\left(e^{-\tau} \delta_{i}\right)\right] \cdot a_{0}$, so, since $\delta_{i} \rightarrow 0$ in $\mathbb{R}$, we get $n_{i} \bar{n}_{i} n_{i}^{\prime} \rightarrow a_{0}$ in $S$. Moreover, $\bar{n}_{i}=\bar{n}\left(\delta_{i}\right) \rightarrow \bar{n}(0)=1_{S}$. By bi-invariance of $f$ under $N$, for all $i$, we have $f\left(n_{i} \bar{n}_{i} n_{i}^{\prime}\right)=f\left(\bar{n}_{i}\right)$, so, passing to the limit, $f\left(a_{0}\right)=f\left(1_{S}\right)$. End of proof of Claim $\alpha$. 
For all $\lambda \in \mathbb{R}$, let $\mathfrak{s}_{\lambda}:=\{A \in \mathfrak{s} \mid(\operatorname{ad} T) A=\lambda A\}$.

Claim $\beta$ : For all $\lambda \in \mathbb{R} \backslash\{0\}$, we have $\mathfrak{s}_{\lambda} \subseteq \mathfrak{m}$. Proof of Claim $\beta$ : Fix $\lambda \in \mathbb{R} \backslash\{0\}$ and $A \in \mathfrak{s}_{\lambda}$. We wish to show that $A \in \mathfrak{m}$.

Fix a sequence $r_{i}$ in $\mathbb{R}$ such that $r_{i} \lambda \rightarrow+\infty$. For all $i$, let $g_{i}:=a\left(r_{i}\right)=\exp \left(r_{i} T\right)$; then, as $A \in \mathfrak{s}_{\lambda}$, we get $(\operatorname{ad} T) A=\lambda A$, so $\left(\operatorname{Ad} g_{i}\right) A=e^{r_{i} \lambda} A$, so $\left(\operatorname{Ad} g_{i}\right)\left(e^{-r_{i} \lambda} A\right)=A$. Moreover, by Claim $\alpha$, we have $g_{i} w=w$. Then, by Lemma 1 (with $v:=w$, with $U_{i}$ replaced by $e^{-r_{i} \lambda} A$ and with $X$ replaced by $A$ ), we are done. End of proof of Claim $\beta$.

Let $\mathfrak{s}_{*}:=(\operatorname{ad} T) \mathfrak{s}$ and let $\mathfrak{s}^{\prime}$ be the Lie subalgebra of $\mathfrak{s}$ generated by the set $\mathfrak{s}_{*}$. By, e.g., Corollary 4.11.7 on p. 130 of $[1], \operatorname{ad} T: \mathfrak{s} \rightarrow \mathfrak{s}$ is real diagonalizable. Then $\mathfrak{s}_{*}=\sum_{\lambda \in \mathbb{R} \backslash\{0\}} \mathfrak{s}_{\lambda}$ and $\mathfrak{s}=\mathfrak{s}_{0}+\mathfrak{s}_{*}$ and, from Claim $\beta$, we get $\mathfrak{s}_{*} \subseteq \mathfrak{m}$. Then $\mathfrak{s}^{\prime} \subseteq \mathfrak{m}$.

The Jacobi identity implies that $\mathfrak{c}_{\mathfrak{s}}(T)$ normalizes the set $(\operatorname{ad} T) \mathfrak{s}$, i.e., that $\mathfrak{s}_{0}$ normalizes $\mathfrak{s}_{*}$. Then $\mathfrak{s}_{0}$ normalizes $\mathfrak{s}^{\prime}$. Moreover, $\mathfrak{s}_{*} \subseteq \mathfrak{s}^{\prime}$, so $\mathfrak{s}_{*}$ normalizes $\mathfrak{s}^{\prime}$. Then $\mathfrak{s}_{0}+\mathfrak{s}_{*}$ normalizes $\mathfrak{s}^{\prime}$, i.e., $\mathfrak{s}$ normalizes $\mathfrak{s}^{\prime}$. That is, $\mathfrak{s}^{\prime}$ is an ideal in $\mathfrak{s}$. Since $T \neq 0$, we have $\operatorname{ad}_{\mathfrak{s}}(T) \neq 0$, so $\mathfrak{s}_{*} \neq\{0\}$, so $\mathfrak{s}^{\prime} \neq\{0\}$. Then, by simplicity of $\mathfrak{s}$, it follows that $\mathfrak{s}^{\prime}=\mathfrak{s}$. Then $\mathfrak{s}=\mathfrak{s}^{\prime} \subseteq \mathfrak{m}$. That is, $S$ fixes $w$. QED

The proof of Claim $\alpha$ above is an algebraic argument derived from the geometry of the proof of Theorem 2.4.2 on p. 29 of [9].

The following is Lemma 4.15 .7 on p. 144 of [1]:

Lemma 7: Let $\mathfrak{g}$ be a Lie algebra with no simple direct summand and let $\mathfrak{n}$ be the nilradical of $\mathfrak{g}$. Then $\mathfrak{c}_{\mathfrak{g}}(\mathfrak{n})=\mathfrak{z}(\mathfrak{n})$.

Proof: Let $\mathfrak{s}$ be a Levi factor of $\mathfrak{g}$ and let $\mathfrak{r}$ be the solvable radical of $\mathfrak{g}$. Then, because $\mathfrak{c}_{\mathfrak{g}}(\mathfrak{n})$ is an ideal in $\mathfrak{g}$, it follows (by, e.g., Lemma 4.10 .19 on p. 119 of [1]) that

$$
\mathfrak{c}_{\mathfrak{g}}(\mathfrak{n}) \quad=\quad\left[\left(\mathfrak{c}_{\mathfrak{g}}(\mathfrak{n})\right) \cap \mathfrak{s}\right]+\left[\left(\mathfrak{c}_{\mathfrak{g}}(\mathfrak{n})\right) \cap \mathfrak{r}\right],
$$

i.e., that $\mathfrak{c}_{\mathfrak{g}}(\mathfrak{n})=\left(\mathfrak{c}_{\mathfrak{s}}(\mathfrak{n})\right)+\left(\mathfrak{c}_{\mathfrak{r}}(\mathfrak{n})\right)$.

By (ii) of Theorem 3.8.3 on p. 206 of $[8]$, we have $[\mathfrak{g}, \mathfrak{r}] \subseteq \mathfrak{n}$. It follows that $[\mathfrak{s}, \mathfrak{r}] \subseteq \mathfrak{n}$, i.e., that ad $: \mathfrak{s} \rightarrow \mathfrak{g l}(\mathfrak{r} / \mathfrak{n})$ is zero. On the other hand, as $\mathfrak{g}$ has no simple direct summand, it follows that ad $: \mathfrak{s} \rightarrow \mathfrak{g l}(\mathfrak{r})$ is faithful. Therefore, by complete reducibility of ad $: \mathfrak{s} \rightarrow \mathfrak{g l}(\mathfrak{r})$, ad $: \mathfrak{s} \rightarrow \mathfrak{g l}(\mathfrak{n})$ is faithful. That is, $\mathfrak{c}_{\mathfrak{s}}(\mathfrak{n})=\{0\}$. Then $\mathfrak{c}_{\mathfrak{g}}(\mathfrak{n})=\left(\mathfrak{c}_{\mathfrak{s}}(\mathfrak{n})\right)+\left(\mathfrak{c}_{\mathfrak{r}}(\mathfrak{n})\right)=\mathfrak{c}_{\mathfrak{r}}(\mathfrak{n})$.

We have $\left[\mathfrak{c}_{\mathfrak{r}}(\mathfrak{n}), \mathfrak{c}_{\mathfrak{r}}(\mathfrak{n})\right] \subseteq[\mathfrak{g}, \mathfrak{r}] \subseteq \mathfrak{n}$ and $\left[\mathfrak{c}_{\mathfrak{r}}(\mathfrak{n}), \mathfrak{n}\right]=\{0\}$. Then $\mathfrak{c}_{\mathfrak{r}}(\mathfrak{n})$ is a nilpotent ideal of $\mathfrak{g}$, so $\mathfrak{c}_{\mathfrak{r}}(\mathfrak{n}) \subseteq \mathfrak{n}$. Then $\mathfrak{c}_{\mathfrak{g}}(\mathfrak{n})=\mathfrak{c}_{\mathfrak{r}}(\mathfrak{n}) \subseteq \mathfrak{n}$, so $\mathfrak{c}_{\mathfrak{g}}(\mathfrak{n})=\left(\mathfrak{c}_{\mathfrak{g}}(\mathfrak{n})\right) \cap \mathfrak{n}=\mathfrak{z}(\mathfrak{n})$. QED

Lemma 8: Let $\mathfrak{g}$ be a Lie algebra with no simple direct summand. Let $\mathfrak{n}$ be the nilradical of $\mathfrak{g}$. Let $\mathfrak{m}$ be a vector subspace of $\mathfrak{g}$. Assume $\forall T \in \mathfrak{m},\left(\mathfrak{c}_{\mathfrak{g}}(T)\right) \cap((\operatorname{ad} T) \mathfrak{g}) \subseteq \mathfrak{m}$. Assume $\exists X \in \mathfrak{m} \backslash\{0\}$ such that ad $X: \mathfrak{n} \rightarrow \mathfrak{n}$ is nilpotent. Then $\mathfrak{m} \cap(\mathfrak{z}(\mathfrak{n}))$ is a nonzero ideal of $\mathfrak{g}$.

Proof: As $\mathfrak{z}(\mathfrak{n})$ is an Abelian ideal of $\mathfrak{g}$, for all $T \in \mathfrak{z}(\mathfrak{n})$, we get $(\operatorname{ad} T) \mathfrak{g} \subseteq\left(\mathfrak{c}_{\mathfrak{g}}(T)\right) \cap(\mathfrak{z}(\mathfrak{n}))$. Then, for all $T \in \mathfrak{m} \cap(\mathfrak{z}(\mathfrak{n}))$, we have $(\operatorname{ad} T) \mathfrak{g} \subseteq\left(\mathfrak{c}_{\mathfrak{g}}(T)\right) \cap((\operatorname{ad} T) \mathfrak{g}) \cap(\mathfrak{z}(\mathfrak{n})) \subseteq \mathfrak{m} \cap(\mathfrak{z}(\mathfrak{n}))$. That is, $\mathfrak{m} \cap(\mathfrak{z}(\mathfrak{n}))$ is an ideal of $\mathfrak{g}$. It remains to show that $\mathfrak{m} \cap(\mathfrak{z}(\mathfrak{n})) \neq\{0\}$.

Define the descending central series of $\mathfrak{n}$ by $\mathfrak{n}^{(1)}:=\mathfrak{n}$ and $\mathfrak{n}^{(i+1)}:=\left[\mathfrak{n}, \mathfrak{n}^{(i)}\right]$. Fix an integer $l \geq 1$ such that $\mathfrak{n}^{(l)}=\{0\}$. Say a sequence $P_{0}, P_{1}, P_{2}, \ldots$ in $\mathfrak{g}$ is good if 
(1) $P_{0} \in \mathfrak{m} \backslash\{0\}$

(2) ad $P_{0}: \mathfrak{n} \rightarrow \mathfrak{n}$ is nilpotent; and

(3) for all integers $i \geq 0$, we have $P_{i+1} \in\left(\mathfrak{c}_{\mathfrak{n}}\left(P_{i}\right)\right) \cap\left(\left(\operatorname{ad} P_{i}\right) \mathfrak{n}\right)$.

By assumption, $X, 0,0,0, \ldots$ is good. For any good sequence $P_{i}$, by induction, for all integers $i \geq 1$, we have $P_{i} \in \mathfrak{n}^{(i)}$; in particular, $P_{l}=0$.

Fix a good sequence $P_{i}$ such that $k:=\max \left\{i \mid P_{i} \neq 0\right\}$ is as large as possible. Then $\left(\mathfrak{c}_{\mathfrak{n}}\left(P_{k}\right)\right) \cap\left(\left(\operatorname{ad} P_{k}\right) \mathfrak{n}\right)=\{0\}$. If $k=0$, then, by $(2), \operatorname{ad}_{\mathfrak{n}}\left(P_{k}\right)$ is nilpotent. If $k \geq 1$, then $P_{k} \in \mathfrak{n}^{(k)} \subseteq \mathfrak{n}$, and so, again, $\operatorname{ad}_{\mathfrak{n}}\left(P_{k}\right)$ is nilpotent.

Then $\left(\mathfrak{c}_{\mathfrak{n}}\left(P_{k}\right)\right) \cap\left(\left(\operatorname{ad} P_{k}\right) \mathfrak{n}\right)$ is intersection of the kernel and image of the nilpotent $\operatorname{map}_{\operatorname{ad}}\left(P_{k}\right)$. However, $\left(\mathfrak{c}_{\mathfrak{n}}\left(P_{k}\right)\right) \cap\left(\left(\operatorname{ad} P_{k}\right) \mathfrak{n}\right)=\{0\}$, while the intersection of the kernel and image of a nonzero nilpotent endomorphism is never $\{0\}$. Then $\operatorname{ad}_{\mathfrak{n}}\left(P_{k}\right)=0$, i.e., $P_{k} \in \mathfrak{c}_{\mathfrak{g}}(\mathfrak{n})$. So, as $\mathfrak{g}$ has no simple direct summand, by Lemma 7 , we get $P_{k} \in \mathfrak{z}(\mathfrak{n})$.

For all integers $i \geq 0$, we have $P_{i+1} \in\left(\mathfrak{c}_{\mathfrak{n}}\left(P_{i}\right)\right) \cap\left(\left(\operatorname{ad} P_{i}\right) \mathfrak{n}\right) \subseteq\left(\mathfrak{c}_{\mathfrak{g}}\left(P_{i}\right)\right) \cap\left(\left(\operatorname{ad} P_{i}\right) \mathfrak{g}\right)$. So, by induction, for all integers $i \geq 0$, we have $P_{i} \in \mathfrak{m}$. Then $0 \neq P_{k} \in \mathfrak{m} \cap(\mathfrak{z}(\mathfrak{n}))$. QED

\section{Decay to zero at Adjoint infinity for connected Lie groups}

Theorem 9: Let $G$ be a connected Lie group acting on a Hilbert space $\mathcal{H}$ by a unitary representation $\pi: G \rightarrow U(\mathcal{H})$. Assume that

(*) no nonzero vector of $\mathcal{H}$ is fixed by a nontrivial normal connected subgroup of $G$.

Let $g_{i}$ be a sequence in $G$ and assume that $\operatorname{Ad}_{\mathfrak{g}}\left(g_{i}\right)$ leaves compact subsets of $\mathrm{GL}(\mathfrak{g})$. Then $\pi\left(g_{i}\right) \rightarrow 0$ in the weak-operator topology on $B(\mathcal{H})$.

\section{Notes:}

1. Condition $(*)$ is satisfied if $G$ is simple and $\mathcal{H}$ admits no $G$-invariant vectors.

2. Condition $(*)$ is satisfied if $\pi$ is faithful and irreducible. (The set of vectors fixed by a normal subgroup is a $G$-invariant subspace. By irreducibility, if such a subspace were nonzero, it would equal $\mathcal{H}$. Then, by faithfulness, the subgroup would be trivial.)

3. I am not sure whether, in Theorem 9, we need that $G$ is connected.

4. One may summarize Theorem 9 as asserting: If a unitary representation of a connected Lie group satisfies $(*)$, then its matrix coefficients decay to zero at "Ad-infinity".

Proof of Theorem 9: Let $B(\mathcal{H})$ have the weak-operator topology. Assume, for a contradiction, that $\pi\left(g_{i}\right) \nrightarrow 0$ in $B(\mathcal{H})$.

Passing to a subsequence, assume that $\left\{\pi\left(g_{i}\right)\right\}$ is bounded away from 0 in $B(\mathcal{H})$. For all $i, \pi\left(g_{i}^{-1}\right)=\left(\pi\left(g_{i}\right)\right)^{*}$, so, because $L \mapsto L^{*}: B(\mathcal{H}) \rightarrow B(\mathcal{H})$ is continuous, we see that $\left\{\pi\left(g_{i}^{-1}\right)\right\}$ is bounded away from 0 in $B(\mathcal{H})$, as well. By (i) of Remark 4 , either $\left\{\operatorname{Ad}_{\mathfrak{g}}\left(g_{i}\right)\right\}$ or $\left\{\operatorname{Ad}_{\mathfrak{g}}\left(g_{i}^{-1}\right)\right\}$ is not precompact in $\operatorname{End}(\mathfrak{g})$. By, if necessary, replacing each $g_{i}$ with $g_{i}^{-1}$, assume that $\left\{\operatorname{Ad}_{\mathfrak{g}}\left(g_{i}\right)\right\}$ is not precompact in $\operatorname{End}(\mathfrak{g})$. Passing to a subsequence, assume that $\operatorname{Ad}_{\mathfrak{g}}\left(g_{i}\right)$ leaves compact subsets of $\operatorname{End}(\mathfrak{g})$. Fix $v \in \mathcal{H}$ such that $g_{i} v \neq 0$ in $\mathcal{H}$. Passing to a subsequence, assume that $g_{i} v \rightarrow w \neq 0$ in $\mathcal{H}$. 
Choose normal connected Lie subgroups $G^{\prime}$ and $G^{\prime \prime}$ of $G$ such that $\mathfrak{g}=\mathfrak{g}^{\prime}+\mathfrak{g}^{\prime \prime}$, such that $\mathfrak{g}^{\prime} \cap \mathfrak{g}^{\prime \prime}=\{0\}$, such that $\mathfrak{g}^{\prime}$ is semisimple or trivial and such that $\mathfrak{g}^{\prime \prime}$ has no simple direct summand. It follows either that $\left\{\operatorname{Ad}_{\mathfrak{g}^{\prime}}\left(g_{i}\right)\right\}$ is not precompact in $\operatorname{End}\left(\mathfrak{g}^{\prime}\right)$ or that $\left\{\operatorname{Ad}_{\mathfrak{g}^{\prime \prime}}\left(g_{i}\right)\right\}$ is not precompact in $\operatorname{End}\left(\mathfrak{g}^{\prime \prime}\right)$.

Case $A:\left\{\operatorname{Ad}_{\mathfrak{g}^{\prime}}\left(g_{i}\right)\right\}$ is not precompact in $\operatorname{End}\left(\mathfrak{g}^{\prime}\right)$. Choose a simple normal connected Lie subgroup $S$ of $G^{\prime}$ such that $\left\{\operatorname{Ad}_{\mathfrak{s}}\left(g_{i}\right)\right\}$ is not precompact in $\operatorname{End}(\mathfrak{s})$. By (ii) of Remark 4, choose $U \in \mathfrak{s}$ such that $\left\{\left(\operatorname{Ad} g_{i}\right) U\right\}$ is not precompact in $\mathfrak{s}$.

Passing to a subsequence, choose $t_{i} \rightarrow 0$ in $\mathbb{R}$ such that $t_{i}\left(\operatorname{Ad} g_{i}\right) U \rightarrow X \neq 0$ in $\mathfrak{s}$. By Remark 3 (with $\mathfrak{g}$ replaced by $\mathfrak{s}$ and $U_{i}$ replaced by $t_{i} U$ ), ad $X: \mathfrak{s} \rightarrow \mathfrak{s}$ is nilpotent. Let $\mathfrak{m}$ be the Lie algebra of $\operatorname{Stab}_{S}(w)$. By Lemma 1 (with $G$ replaced by $S$ and $U_{i}$ replaced by $\left.t_{i} U\right)$, we see that $X \in \mathfrak{m}$. Then, by Proposition $6, S$ fixes $w$. However, $S$ is normal in $G^{\prime}$, and therefore in $G$, contradicting (*). End of Case $A$.

Case B: $\left\{\operatorname{Ad}_{\mathfrak{g}^{\prime \prime}}\left(g_{i}\right)\right\}$ is not precompact in $\operatorname{End}\left(\mathfrak{g}^{\prime \prime}\right)$. By (ii) of Remark 4, choose $U \in \mathfrak{g}^{\prime \prime}$ such that $\left\{\left(\operatorname{Ad} g_{i}\right) U\right\}$ is not precompact in $\mathfrak{g}^{\prime \prime}$.

Passing to a subsequence, choose $t_{i} \rightarrow 0$ in $\mathbb{R}$ such that $t_{i}\left(\operatorname{Ad} g_{i}\right) U \rightarrow X \neq 0$ in $\mathfrak{g}^{\prime \prime}$. By Remark 3 (with $\mathfrak{g}$ replaced by $\mathfrak{g}^{\prime \prime}$ and $U_{i}$ replaced by $t_{i} U$ ), ad $X: \mathfrak{g}^{\prime \prime} \rightarrow \mathfrak{g}^{\prime \prime}$ is nilpotent. Let $\mathfrak{m}$ be the Lie algebra of $\operatorname{Stab}_{G^{\prime \prime}}(w)$. By Lemma 1 (with $G$ replaced by $G^{\prime \prime}$ and $U_{i}$ replaced by $t_{i} U$ ), we get $X \in \mathfrak{m}$. By Corollary 2 (with $G$ replaced by $G^{\prime \prime}$ ), for all $T \in \mathfrak{m}$, we have $\left(\mathfrak{c}_{\mathfrak{g}^{\prime \prime}}(T)\right) \cap\left((\operatorname{ad} T) \mathfrak{g}^{\prime \prime}\right) \subseteq \mathfrak{m}$. Let $\mathfrak{n}$ be the nilradical of $\mathfrak{g}^{\prime \prime}$. Then ad $X: \mathfrak{n} \rightarrow \mathfrak{n}$ is nilpotent. Then, by Lemma 8 (with $\mathfrak{g}$ replaced by $\left.\mathfrak{g}^{\prime \prime}\right), \mathfrak{m} \cap\left(\mathfrak{z}(\mathfrak{n})\right.$ ) is a nonzero ideal of $\mathfrak{g}^{\prime \prime}$, and therefore of $\mathfrak{g}$. Then the connected Lie subgroup of $G$ corresponding to $\mathfrak{m} \cap(\mathfrak{z}(\mathfrak{n}))$ is a nontrivial normal connected subgroup of $G$ fixing $w$, contradicting $(*)$. End of Case B. QED

Thanks to D. Witte Morris for help in developing the following example:

Example 10: In the statement of Theorem 9, $(*)$ cannot be replaced by

$\left(*^{\prime}\right)$ no nonzero vector of $\mathcal{H}$ is fixed by a noncompact normal connected subgroup of $G$ even under the assumption that $\pi$ is faithful.

Proof: Let $H$ be the 3-dimensional Heisenberg group, and let $Z$ denote the center of $H$. Let $\phi: H \rightarrow \mathbb{R}^{2}$ be a surjective homomorphism whose kernel is $Z$, and let $D$ be a nontrivial discrete subgroup of $Z$. Let $G:=H / D$ and let $p: H \rightarrow G$ be the canonical homomorphism. Let $\psi: G \rightarrow \mathbb{R}^{2}$ be defined by $\psi(p(g))=\phi(g)$.

Let $\mathcal{H}^{\prime}$ be a Hilbert space and let $\rho: \mathbb{R}^{2} \rightarrow U\left(\mathcal{H}^{\prime}\right)$ be a unitary representation of $\mathbb{R}^{2}$ with no nonzero invariant vectors such that not all matrix coefficients decay to zero at infinity. Let $\pi^{\prime}:=\rho \circ \psi: G \rightarrow U\left(\mathcal{H}^{\prime}\right)$. Let $\mathcal{H}^{\prime \prime}$ be another Hilbert space and fix a faithful unitary representation $\pi^{\prime \prime}: G \rightarrow U\left(\mathcal{H}^{\prime \prime}\right)$.

Let $\mathcal{H}:=\mathcal{H}^{\prime} \oplus \mathcal{H}^{\prime \prime}$. Then $\pi:=\pi^{\prime} \oplus \pi^{\prime \prime}: G \rightarrow U(\mathcal{H})$ is faithful and satisfies $\left(*^{\prime}\right)$, but does not enjoy the property that all matrix coefficients decay to zero at Ad-infinity. QED

\section{Decay to zero at projective infinity for algebraic groups}

Theorem 11: Let $G$ be the connected real points of a linear algebraic $\mathbb{R}$-group. Let $\pi: G \rightarrow U(\mathcal{H})$ be an irreducible unitary representation on a Hilbert space $\mathcal{H}$. Then 
$\pi(g) \rightarrow 0$ in the weak-operator topology on $B(\mathcal{H})$, as $g$ leaves compact subsets of $G$ modulo the projective kernel of $\pi$.

Proof: Let $K:=\operatorname{ker}(\pi)$. Let $\bar{G}:=G / K$. Let $p: G \rightarrow \bar{G}$ be the canonical homomorphism. For all $g \in G$, let $\bar{g}:=p(g)$. Define $\bar{\pi}: \bar{G} \rightarrow U(\mathcal{H})$ by $\bar{\pi}(\bar{g})=\pi(g)$.

The Adjoint representation $\mathrm{Ad}: G \rightarrow \mathrm{GL}(\mathfrak{g})$ is algebraic and $\mathfrak{k}$ is an invariant subspace, so Ad : $G \rightarrow \operatorname{GL}(\mathfrak{g} / \mathfrak{k})$ is algebraic and therefore has closed image. So, since $\overline{\mathfrak{g}}=\mathfrak{g} / \mathfrak{k}$ and since $\operatorname{Ad}_{\bar{g}}(\bar{G})=\operatorname{Ad}_{\overline{\mathfrak{g}}}(G)$, we see that $\operatorname{Ad}_{\overline{\mathfrak{g}}}(\bar{G})$ is closed in $\operatorname{GL}(\overline{\mathfrak{g}})$. Then $\operatorname{Ad}: \bar{G} \rightarrow \operatorname{GL}(\overline{\mathfrak{g}})$ factors to a proper, injective Lie group homomorphism $F: \bar{G} /(Z(\bar{G})) \rightarrow \operatorname{GL}(\overline{\mathfrak{g}})$.

Assume that $g_{i}$ leaves compact subsets of $\mathrm{G}$ modulo the projective kernel of $\pi$. We wish to show that $\pi\left(g_{i}\right) \rightarrow 0$ in the weak-operator topology on $U(\mathcal{H})$.

The sequence $\overline{g_{i}}$ leaves compact subsets of $\bar{G}$ modulo the projective kernel of $\bar{\pi}$. By Schur's Lemma, since $\bar{\pi}$ is faithful and irreducible, its projective kernel is $Z(\bar{G})$. So, since $F$ is proper, $\operatorname{Ad} \overline{g_{i}}$ leaves compact subsets of $\mathrm{GL}(\overline{\mathfrak{g}})$. Then, by Theorem $9, \bar{\pi}\left(\overline{g_{i}}\right) \rightarrow 0$ in the weak-operator topology on $U(\mathcal{H})$. For all $i, \bar{\pi}\left(\overline{g_{i}}\right)=\pi\left(g_{i}\right)$, so we are done. QED

The proof of Theorem 11 also works for any connected Lie group that is locally isomorphic to a real linear algebraic group.

\section{References}

[1] S. Adams: Dynamics on Lorentz Manifolds, World Scientific, Singapore, 2001.

[2] R. Ellis and M. Nerurkar: Weakly almost periodic flows, Trans. Amer. Math. Soc. 313 no. 1 (1989), 103-119.

[3] S. Helgason: Differential Geometry, Lie Groups and Symmertic Spaces, Academic Press, London, 1978.

[4] R. Howe and C. C. Moore: Asymptotic properties of unitary representations., J. Funct. Anal. 32 no. 1 (1979), 72-96.

[5] N. Kowalsky: oncompact simple automorphism groups of Lorentz manifolds and other geometric manifolds, Ann. of Math. (2) 144 no. 3 (1996), 611-640.

[6] C. C. Moore: Ergodicitiy of flows on homogeneous spaces, Amer. J. Math. 88 (1966), $154-178$.

[7] T. Sherman: A weight theory for unitary representations, Canad. J. Math. 18 (1966), $159-168$.

[8] V. S. Varadarajan: Lie Groups, Lie Algebras, and Their Representations, SpringerVerlag, New York, 1984.

[9] R. J. Zimmer: Ergodic Theory and Semisimple Groups, Birkhäuser, Boston, 1984. 